\title{
Increased incidence of Mycoplasma pneumoniae infection in Finland, 2010-2011
}

A Polkowska (aleksandra.polkowska@thl.fi) ${ }^{1,2}$, A Harjunpää ${ }^{1}$, S Toikkanen ${ }^{1}$, M Lappalainen $^{3}$, R Vuento ${ }^{4}$, T Vuorinen ${ }^{5}$, J Kauppinen ${ }^{6}$, H Flinck ${ }^{7}$, 0 Lyytikäinen ${ }^{1}$

1. National Institute for Health and Welfare (THL), Department of Infectious Disease Surveillance and Control, Helsinki, Finland

2. European Programme for Intervention Epidemiology Training (EPIET), Stockholm, Sweden

3. Helsinki University Hospital, Laboratory Services (HUSLAB), Department of Virology and Immunology, Helsinki, Finland

4. Fimlab Laboratories, Pirkanmaa Hospital District, Tampere, Finland

5. Department of Virology, University of Turku, Turku, Finland

6. Eastern Finland Laboratory Centre Joint Authority Enterprise (ISLAB), Kuopio, Finland

7. United Medix Laboratories Ltd, Espoo, Finland

Citation style for this article:

Polkowska A, Harjunpää A, Toikkanen S, Lappalainen M, Vuento R, Vuorinen T, Kauppinen J, Flinck H, Lyytikäinen O. Increased incidence of Mycoplasma

pneumoniae infection in Finland, 2010-2011.

Euro Surveill. 2012;17(5):pii=20072. Available online: http://www.eurosurveillance.org/ViewArticle.aspx?Articleld=20072

Article published on 2 February 2012

The number of cases of Mycoplasma pneumoniae infection detected by laboratory-based surveillance increased in Finland in late 2010. During 2011, the number of cases was four times higher than during the previous epidemic in 2005. The 2011 epidemic affected mostly school-age children. The increased number of cases was probably not due to changes in laboratory procedures, but public interest may have had an effect, since the number of Google queries followed closely the epidemic curve.

The number of cases of Mycoplasma pneumoniae infection in Finland started to increase in October 2010 (222 cases; 4.1 per 100,000 population) and rose further during 2011 (in October, 1,242 cases; 23.1 cases per 100,000 population). Denmark, England and Wales also saw an increased incidence of $M$. pneumoniae infections in late $2010[1,2]$. Throughout 2011, the epidemic of $M$. pneumoniae infection in Finland attracted considerable public interest and media attention.

In order to assess the extent of this ongoing epidemic, we analysed the data on $M$. pneumoniae infection from laboratory-based surveillance. We also evaluated whether changes in laboratory methods and practices as well as public interest in the epidemic during 2011 were related to the size of the epidemic.

\section{Background}

M. pneumoniae causes mainly infection of the upper respiratory tract (tracheitis, bronchitis) and, in 3-10\% of cases, pneumonia. Rare neurological symptoms such as meningitis and Guillain-Barré syndrome can be observed [3]. The bacterium is spread by respiratory droplets and direct contact with an infected person. The disease occurs in all age groups but is most common among children aged 7-16 years and young adults aged 17-25 years. Presumably due to lack of lifelong protective immunity and changes in circulating M. pneumoniae strains, epidemics typically occur in 3-5-year intervals [3], with seasonal peaks in autumn and winter.

\section{National laboratory-based surveillance system}

The laboratory-based surveillance system in Finland (population 5.4 million) covers 20 healthcare districts with catchment populations ranging from 68,000 to 1.4 million. Since 1995 , all clinical microbiology laboratories mandatorily notify all positive findings of M. pneumoniae (culture, diagnostic rise in M. pneumoniae-specific IgG antibody titre, detection of $M$. pneumoniae-specific IgM antibodies and nucleic acid detection) to the National Infectious Disease Register, maintained by the National Institute for Health and Welfare. The following information is collected with each notification: date of birth, sex, unique national identity code, place of treatment, type of specimen and diagnostic method. Multiple notifications with the same national identity code are merged into one case, if reported within 12 months of each other. In this study, we analysed cases of $M$. pneumoniae infection notified to the National Infectious Disease Register from 1 January 1995 to 31 December 2011.

\section{Study approach}

To investigate whether there have been changes in laboratory methods or practices regarding $M$. pneumoniae diagnosis, we carried out an email survey of the five biggest laboratories in the country, located in Helsinki, Turku, Tampere and Kuopio, which notified $97.5 \%$ of all M. pneumoniae cases during 2010 and 2011. We asked about the total number of tests performed per month and the proportion of tests positive for M. pneumoniae per month in 2010 and 2011. In addition, we asked the laboratories which tests they used and whether there 
had been changes in tests since the previous epidemic in 2005 .

To investigate the extent of public interest in M. pneumoniae, we used Google Insight for Search beta and Google AdWords applications. We obtained the number of Google queries for 'mycoplasma' in Finland, during 2004 to 2011 by month.

\section{Surveillance data}

The number of cases of $M$. pneumoniae infection began to increase since October 2010 (Figure 1). The first peak was in March $2011(n=838)$. The number of cases dropped between April and July 2011 and then started to increase again in September $2011(n=667)$. The number of cases rose from 1,948 (36.2 per 100,000 population) in 2010 to 7,772 (145 per 100,000 population) in 2011. In 2011, the increase in the number of M. pneumoniae cases was detected in all healthcare districts but the incidence varied regionally (range by healthcare district: 55 per 100,000 population to 257 per 100,000 population).

During 1995 to 2011, a total of 22,835 cases were notified. Previous epidemics occurred in the winters of 2000-2002 and 2004-2006 with a peak in 2005 ( 1,881 cases; 36 per 100,000 population). These earlier epidemics lasted about two years, i.e. over two cold seasons.

The annual incidence during 1995 to 2011 was highest among children aged 5-14 years and lowest among elderly persons aged 65 years and older (Figure 2).

In 2011, the median age of the cases was 18 years (range: $0-85$ ) and 4,418 (57\%) were female. During
2005 to 2011, the median age of the cases was also 18 years (range: $0-104)$ and 13,185 (58\%) were female. The difference by sex was most prominent in persons aged 15-64 years, among whom the incidence was 1.8fold higher in females than in males both during 1995 to 2010 and in 2011.

Most of the notifications were based on testing of serum or plasma $(22,486 ; 98.5 \%)$, a few were from bronchoalveolar lavage $(63 ; 0.3 \%)$, pharyngeal or nasopharyngeal swabs $(94 ; 0.4 \%)$ or cerebrospinal fluid $(35 ; 0.2 \%)$. In $98 \%$ of the notifications, the diagnostic method was detection of $M$. pneumoniae-specific antibodies; the rest were based on nucleic acid detection by PCR.

\section{Laboratory survey}

In the five laboratories taking part in the survey, detection of M. pneumoniae was mainly based on serological tests by enzyme immune assay (EIA). Diagnosis of infection required a diagnostic rise in $M$. pneumoniae-specific IgG antibody titre and/or detection of a M. pneumoniae-specific IgM. If necessary, the laboratory recommended collecting convalescent paired sera. Since the previous epidemic in 2005 , there has been no change in diagnostic methods.

The number of serological tests performed for M. pneumoniae in the five laboratories was on average nearly four times higher in 2011 than in 2010 (range of increase by laboratory: 200-500\%). The proportion of tests positive for M. pneumoniae during 2010 and 2011 varied between $8 \%$ and $17 \%$ in the five laboratories. There was also variation during 2010 and 2011 in four of the laboratories: in three the proportion of positive tests increased (from $8 \%$ to $9 \%$, from $9 \%$ to $11 \%$, from

\section{FIGURE 1}

Cases of Mycoplasma pneumoniae infection by month reported to the National Infectious Diseases Register, Finland, 1995-2011

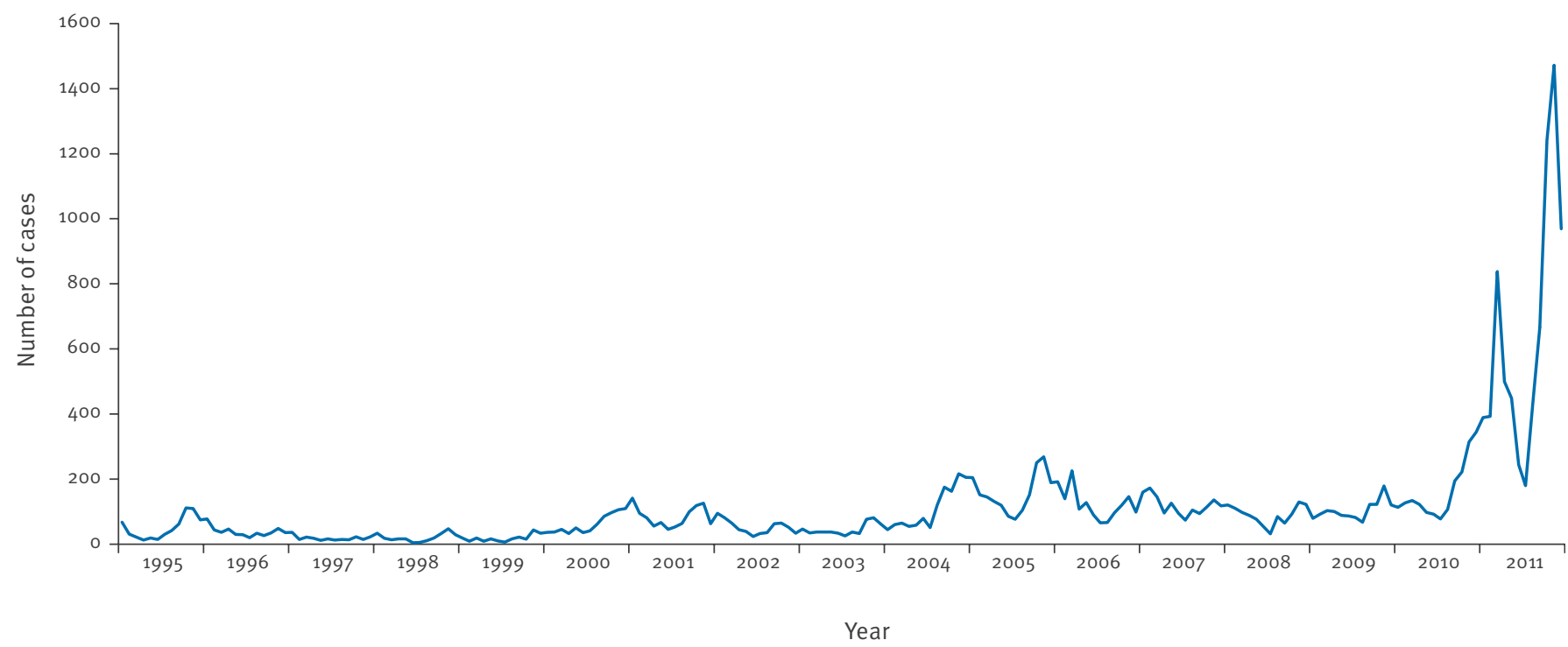


$11 \%$ to $17 \%$ ); in one it decreased slightly (from $8.5 \%$ to $8.1 \%)$ and in one, it remained the same.

\section{Public interest, assessed \\ through Google queries}

The first two peaks in the number of Google queries for 'mycoplasma' occurred during the epidemics in 2004-2005 and 2005-2006. After 2007, the number was stable. In October 2010, however, it rose again, peaking in March and November 2011 (Figure 3). As described in [4], the numbers of Google queries in Figure 3 reflect the number of searches per month for 'mycoplasma' relative to the total number of searches on Google between 2004 and 2011 in Finland. The data are normalised (data are divided by a common variable to cancel out the variable's effect on the data) and presented on a scale from o to 100. On the basis of data from Google AdWords, the approximate 12-month mean number of Google queries for 'mycoplasma' in Finland amounted to $7.3 \%$ of global searching for this term in 2011. Data on global and local searches in the previous years were not available.

\section{Discussion}

Our study based on nationwide laboratory data showed a fourfold increase in incidence and number of cases of $M$. pneumoniae infection in 2011 compared with the previous epidemic in 2005 - the highest in the history of our national surveillance. In Denmark, England and Wales, the previous epidemics were larger than their current ones (at the start of the current epidemics) $[1,2]$. There were no major changes in laboratory

\section{FIGURE 2}

Annual incidence of Mycoplasma pneumoniae infection per 100,000 population by age group reported to the National Infectious Diseases Register, Finland, 1995-2011

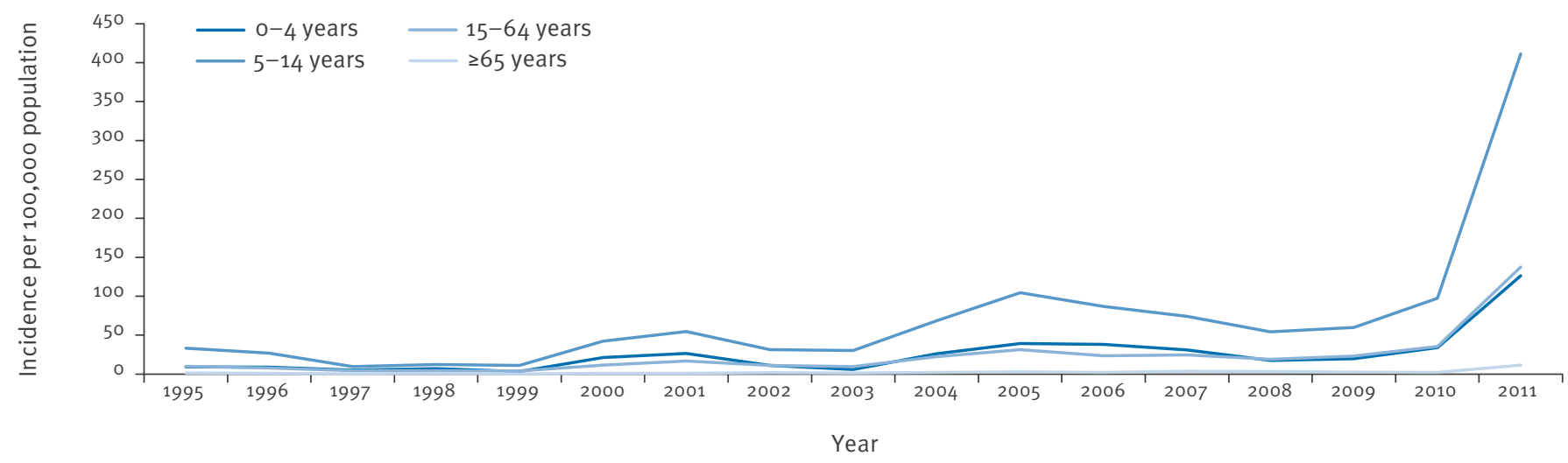

Source: National Infectious Diseases Register, Finland.

\section{FIGURE 3}

Cases of Mycoplasma pneumoniae by month reported to the National Infectious Diseases Register and 'mycoplasma' queries in Google, Finland, 2004-2011

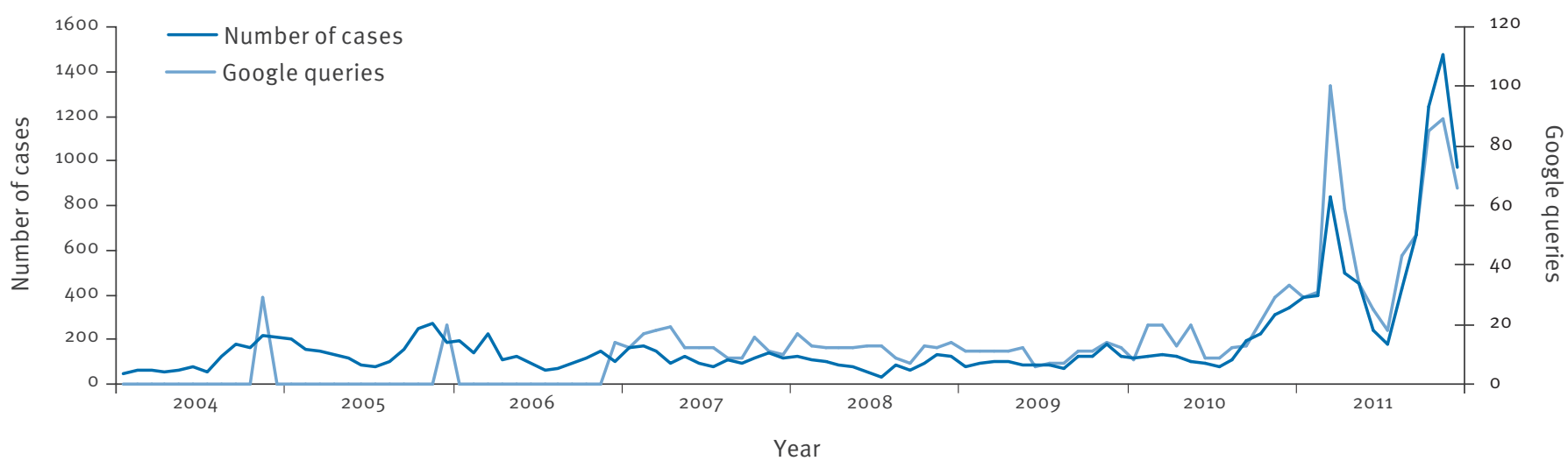

The Google queries shown in the graph do not represent absolute search volume numbers, because the data are normalised and scaled from 0 to 100 . Normalisation means that data sets are divided by an unrelated, common Web search query. Data are scaled using the average search volume over the selected time period as a denominator.

Source: National Infectious Diseases Register, Google Insights for Search (Google data downloaded 21 December 2011 ). 
diagnostics that could have contributed to the extent of the epidemic in Finland. However, data on the number of tests carried out from 2005 to 2006 were not available. As the number of tests performed may influence the rate of positive results, comparison of the heights of the epidemic peaks should therefore be made with caution.

Google is known to be a popular information source [5]. In Finland, Internet access is widespread: about $89 \%$ of the population aged $16-74$ years used the Internet in the past three months [6]. On the basis of our results, we can assume that the high number of cases of M. pneumoniae infection - especially during the current epidemic - may partly reflect the intense public interest in and awareness of the disease. Patients with a prolonged cough may have been more active than in the past in seeking care and requesting testing for Mycoplasma, which may, in some instances, have lead to unnecessary antimicrobial treatment as prolonged cough after the acute phase of infection may not benefit from such treatment.

Diagnostic testing for $M$. pneumoniae also rose around fourfold in 2011, compared with 2010. The variation in proportion of tests positive for $M$. pneumoniae between laboratories $(8-17 \%)$ could be related to differences in interpreting the serological results. This finding needs further evaluation, but highlights the importance of standardisation of laboratory methodology. It may also be a sign of regional differences in diagnostic activity and case ascertainment, since the sampling was not structured for epidemiological surveillance. Laboratory diagnosis of $M$. pneumoniae infection is not easy. High levels of $M$. pneumoniae-specifc IgM antibodies can persist for several weeks to up to one year after an acute infection $[3,7,8]$. Furthermore, $M$. pneumoniaespecifc IgG antibodies may remain elevated up to four years after illness [9]. In addition, it may be difficult for clinical microbiologists to interpret borderline results, since the date of symptom onset is rarely available in the laboratories.

Our survey found that PCR was not widely used in Finland for diagnosis of $M$. pneumoniae infection. PCR has been found to be superior to serology for the diagnosis of acute $M$. pneumoniae infection and has been shown to be highly sensitive, specific and rapid [10]. However, a positive PCR may be a sign of transient asymptomatic carriage of $M$. pneumoniae or the persistence of the pathogen after infection [9]. In Denmark, where PCR-based surveillance for $M$. pneumoniae infections is established, the proportion of tests positive for $M$. pneumoniae was approximately $3 \%$ since 2007 until it rose to $15 \%$ in September 2010 when the current epidemic started [1].

We also found that culturing of $M$. pneumoniae was also scarce in Finland. It is known to be difficult, timeconsuming and expensive, and therefore rarely routinely used in clinical practice [11]. Thus, information on the molecular epidemiology of circulating $M$. pneumoniae strains is lacking, and it is also not known whether the current epidemic strains are sensitive or resistant to macrolides, the antimicrobials commonly used in treatment [3].

Since our study was based on laboratory data only, we did not have information on clinical manifestation, severity of the disease or treatment. The burden of the M. pneumoniae epidemic in Finland remains unknown. Although people with $M$. pneumoniae infections are mainly seen as outpatients, a register-based linkage study between laboratory-confirmed cases and hospitalisation data or a time series of pneumonia-associated hospitalisation rates could give an insight into the burden and use of macrolides could be analysed.

Physicians and the public have been informed about the symptoms and treatment of Mycoplasma infections, as well as the difficulties in diagnosis.

References

1. Rasmussen JN, Voldstedlund M, Andersen RL, EllermannEriksen S, Jensen TG, Johansen HK, et al. Increased incidence of Mycoplasma pneumoniae infections detected by laboratory-based surveillance in Denmark in 2010. Euro Surveill. 2010;15(45):pii=19708. Available from: http://www. eurosurveillance.org/ViewArticle.aspx?Articleld=19708

2. Chalker VJ, Stocki T, Mentasti M, Fleming D, Harrison TG. Increased incidence of Mycoplasma pneumoniae infection in England and Wales in 2010: multiocus variable number tandem repeat analysis typing and macrolide susceptibility. Euro Surveill. 2011;16(19):pii=19865. Available from: http://www. eurosurveillance.org/ViewArticle.aspx?Articleld=19865

3. Atkinson TP, Balish MF, Waites KB. Epidemiology, clinical manifestations, pathogenesis and laboratory detection of Mycoplasma pneumoniae infections. FEMS Microbiol Rev. 2008;32(6):956-73.

4. Google. What do the numbers on the graph mean? Insights for search. Mountain View, CA: Google. [Accessed 22 Dec 2011]. Available from: http://support.google.com/insights/bin/ answer.py?hl=en-GB\&answer $=87285$

5. Ginsberg J, Mohebbi MH, Patel RS, Brammer L, Smolinski MS, Brilliant L. Detecting influenza epidemics using search engine query data. Nature. 2009;457(7232):1012-4.

6. Statistics Finland. Use of information and communications technology. Helsinki: Statistics Finland. [Accessed 12 Jan 2012]. Available from: http://www.stat.fi/til/sutivi/ index_en.html

7. Thurman KA, Walter ND, Schwartz SB, Mitchell SL, Dillon MT, Baughman AL, et al. Comparison of laboratory diagnostic procedures for detection of Mycoplasma pneumoniae in community outbreaks. Clin Infect Dis. 2009;48(9):1244-9.

8. Vikerfors TG, Brodin G, Grandien M, Hirschberg L, Krook A, Petterson CA. Detection of specific IgM antibodies for the diagnosis of Mycoplasma pneumoniae infections: a clinical evaluation. Scand J Infect Dis.1988;20(6):601-10.

9. Daxboeck F, Krause R, Wenisch C. Laboratory diagnosis of Mycoplasma pneumoniae infection. Clin Microbiol Infect. 2003;9(4):263-73.

10. Beersma MF, Dirven K, van Dam AP, Templeton KE, Claas EC, Goossens H. Evaluation of 12 commercial tests and the complement fixation test for Mycoplasma pneumoniae-specific immunoglobulin G (IgG) and IgM antibodies, with PCR used as the "gold standard". J Clin Microbiol. 2005;43(5):2277-85.

11. Sánchez-Vargas FM. Gómez-Duarte OG. Mycoplasma pneumoniae-an emerging extra-pulmonary pathogen. Clin Microbiol Infect 2008;14(2):105-17. 